\title{
MXenes for Plasmonic Photodetection
}

\author{
Dhinesh Babu Velusamy, Jehad K. El-Demellawi, Ahmed M. El-Zohry, Andrea Giugni, \\ Sergei Lopatin, Mohamed N. Hedhili, Ahmed E. Mansour, Enzo Di Fabrizio, \\ Omar F. Mohammed, and Husam N. Alshareef*:
}

MXenes have recently shown impressive optical and plasmonic properties associated with their ultrathin-atomic-layer structure. However, their potential use in photonic and plasmonic devices has been only marginally explored. Photodetectors made of five different $\mathrm{MXenes}$ are fabricated, among which molybdenum carbide MXene $\left(\mathrm{Mo}_{2} \mathrm{CT}_{x}\right)$ exhibits the best performance. $\mathrm{Mo}_{2} \mathrm{CT}_{x}$ MXene thin films deposited on paper substrates exhibit broad photoresponse in the range of 400-800 nm with high responsivity (up to $9 \mathrm{~A} \mathrm{~W}^{-1}$ ), detectivity $\left(\approx 5 \times 10^{11}\right.$ Jones), and reliable photoswitching characteristics at a wavelength of $660 \mathrm{~nm}$. Spatially resolved electron energy-loss spectroscopy and ultrafast femtosecond transient absorption spectroscopy of the MXene nanosheets reveal that the photoresponse of $\mathrm{Mo}_{2} \mathrm{CT}_{x}$ is strongly dependent on its surface plasmon-assisted hot carriers. Additionally, $\mathrm{Mo}_{2} \mathrm{CT}_{x}$ thin-film devices are shown to be relatively stable under ambient conditions, continuous illumination and mechanical stresses, illustrating their durable photodetection operation in the visible spectral range. Micro-Raman spectroscopy conducted on bare $\mathrm{Mo}_{2} \mathrm{CT}_{x}$ film and on gold electrodes allowing for surface-enhanced Raman scattering demonstrates surface chemistry and a specific low-frequency band that is related to the vibrational modes of the single nanosheets. The specific ability to detect and excite individual surface plasmon modes provides a viable platform for various MXene-based optoelectronic applications.

"A" is an A-group element, e.g., $\mathrm{Al}, \mathrm{Ga}$, $\mathrm{Si}$, etc., " $\mathrm{X}$ " is carbon and/or nitrogen, and $\mathrm{T}$ denotes the surface-terminated functional groups such as $-\mathrm{OH},-\mathrm{F}$, and $-\mathrm{O},{ }^{[1]}$ and $x$ in $\mathrm{T}_{x}$ represents the population of those surface-terminated moieties. Thus far, owing to their promising electrochemical characteristics, this new class of 2D materials has been predominantly used in energy conversion and storage applications..$^{[2,3,12,13]}$ Nonetheless, they have also endowed fascinating prospects for a variety of emerging applications in electronics, sensing, and photonics..$^{[2,14-16]}$ In this regard, MXenes have recently exhibited impressive optical properties, e.g., tunable broadband absorption ${ }^{[16-18]}$ and intense surface plasmon excitations, ${ }^{[19,20]}$ underlining their potential for optoelectronic and plasmonic applications. However, theoretical ${ }^{[20]}$ and experimental investigations ${ }^{[16,21,22]}$ in this context remain sparse and mainly limited to the most studied $\mathrm{Ti}_{3} \mathrm{C}_{2} \mathrm{~T}_{x}$ MXene.

In this work, five different MXene nanosheets, i.e., $\mathrm{Mo}_{2} \mathrm{CT}_{x}, \mathrm{Ti}_{3} \mathrm{C}_{2} \mathrm{~T}_{x}, \mathrm{Nb}_{2} \mathrm{CT}_{x}$, $\mathrm{T}_{2} \mathrm{CT}_{x}$, and $\mathrm{V}_{2} \mathrm{CT}_{x}$, were first synthesized

MXenes, a thriving family of transition metal-based 2D materials, have garnered significant interest due to their intriguing physicochemical properties and solution processability. ${ }^{[1-11]} 2 \mathrm{D}$ MXene nanosheets are obtained by selectively etching away the A atoms from their parent ternary "MAX" phases constituting a large family of layered transition metal carbides, nitrides, or carbonitrides. The synthesized 2D MXenes have the general formula: $\mathrm{M}_{n+1} \mathrm{X}_{n} \mathrm{~T}_{x}$, where " $\mathrm{M}$ " is an early transition metal,

Dr. D. B. Velusamy, J. K. El-Demellawi, Dr. A. M. El-Zohry, Dr. A. Giugni,

Dr. A. E. Mansour, Prof. E. Di Fabrizio, Prof. O. F. Mohammed,

Prof. H. N. Alshareef

Physical Science and Engineering Division

King Abdullah University of Science and Technology (KAUST)

Thuwal 23955-6900, Kingdom of Saudi Arabia

E-mail: husam.alshareef@kaust.edu.sa

Dr. S. Lopatin, Dr. M. N. Hedhili

Core Labs

King Abdullah University of Science and Technology (KAUST)

Thuwal 23955-6900, Kingdom of Saudi Arabia

The ORCID identification number(s) for the author(s) of this article can be found under https://doi.org/10.1002/adma.201807658.

DOI: 10.1002/adma.201807658 from their parent MAX phases (see the Experimental Section in the Supporting Information for the detailed synthesis of MAX and MXenes). Their structural, morphological, and steadystate optical characteristics are displayed in Figures S1-S6 in the Supporting Information. Among the five studied MXenes, $\mathrm{Mo}_{2} \mathrm{CT}_{x}$ was selected as a model system for its relative stability against oxidation, moderately high free carrier density, and electrical conductivity, ${ }^{[7]}$ though not as high as $\mathrm{Ti}_{3} \mathrm{C}_{2} \mathrm{~T}_{x}{ }^{[2,18,19]}$ In principle, for the sake of not having high dark currents, materials with metal-like conductivity are not preferred for photodetectors.

In this regard, for the first time, we demonstrate $\mathrm{Mo}_{2} \mathrm{CT}_{x}$ thin film photodetectors that operate in the visible spectral region. The optoelectronic properties of the mechanically flexible arrays of two-terminal, parallel-type photodetectors based on $\mathrm{Mo}_{2} \mathrm{CT}_{x}$ MXene thin films deposited on paper substrates (nylon membrane filters) are thoroughly investigated. We show that the responsivity and detectivity of the $\mathrm{Mo}_{2} \mathrm{CT}_{x}$ photodetectors can reach as high as $9 \mathrm{~A} \mathrm{~W}^{-1}$ and $\approx 5 \times 10^{11}$ Jones, respectively, at a wavelength of $660 \mathrm{~nm}$. It is worth mentioning that the responsivity of the $\mathrm{Mo}_{2} \mathrm{CT}_{x}$ MXene devices we propose is $\approx 18000$ and $\approx 1200$ times higher than the first reported graphene ${ }^{[23]}$ 
and $\mathrm{MoS}_{2}{ }^{[24]}$ photodetectors, respectively. We found that the photocurrent in $\mathrm{Mo}_{2} \mathrm{CT}_{x}$ is principally controlled by efficient surface plasmon-assisted hot electron generation. The MXene films, composed of the disordered superposition of crystalline nanosheets with dimensions about hundreds of nanometers, offer naturally high density of edges and nanometric gaps that can efficiently relax plasmonic momentum constraints promoting energized hot electrons generation. ${ }^{[25]}$ The relatively lower electronic density of $\mathrm{Mo}_{2} \mathrm{CT}_{x}$ (i.e., $\approx 3 \times 10^{20} \mathrm{~cm}^{-3}$ ), ,7] with respect to conventional plasmonic metals, allows for a longer electronic mean free path, supporting a rather long experimental electron-electron scattering relaxation time and the very good performances reported here. The existence and the distribution of a variety of surface plasmon modes over individual $\mathrm{Mo}_{2} \mathrm{CT}_{x}$ nanosheets are visualized by the combination of scanning transmission electron microscopy (STEM) and ultrahigh resolution electron energy loss spectroscopy (EELS). The dynamics of the generated plasmon-assisted hot carriers upon excitation is elucidated using femtosecond visible transient absorption experiments. We also show that $\mathrm{Mo}_{2} \mathrm{CT}_{x}$-based devices have maintained over $80 \%$ of the initial photocurrent generation efficiency even after $12000 \mathrm{~s}$ of continuous illumination of $660 \mathrm{~nm}$ light. While the $I_{\mathrm{on}} / I_{\text {off }}$ ratio remained almost the same over the course of $30+$ days of storage under vacuum at room temperature. Moreover, the arrays of $\mathrm{Mo}_{2} \mathrm{CT}_{x}$ thin film photodetectors are shown to be readily bendable under multiple and repeated deformations.

Figure 1a shows a schematic representation of a $\mathrm{Mo}_{2} \mathrm{CT}_{x}$ nanosheet, where one layer of carbon is sandwiched between two layers of molybdenum, while the surface of the nanosheet is randomly terminated with functional surface moieties, i.e., $-\mathrm{OH},-\mathrm{O}$, and $-\mathrm{F}$. The transmission electron microscopy (TEM) image and corresponding selected area electron diffraction (SAED) pattern of $\mathrm{Mo}_{2} \mathrm{CT}_{x}$ nanosheets show a smooth surface morphology with a high crystal quality (Figure 1b). Further, the atomic-resolution scanning transmission electron microscopy image of $\mathrm{Mo}_{2} \mathrm{CT}_{x}$ nanosheets (Figure 1c) indicates the hexagonal structure of the basal planes without obvious nanometer-scale defects or carbide amorphization. ${ }^{[8]}$ The UV-vis-NIR absorption spectra of as-prepared suspension of $\mathrm{Mo}_{2} \mathrm{CT}_{x}$ nanosheets in water $(0.6 \mathrm{mg} \mathrm{mL}-1$, red) and a $\approx 1.8 \mu \mathrm{m}$ thick film of $\mathrm{Mo}_{2} \mathrm{CT}_{x}$ (blue) are depicted in Figure $1 \mathrm{~d}$. The difference between the absorption of film and suspension is attributed to light scattering by the suspended colloidal $\mathrm{Mo}_{2} \mathrm{CT}_{x}$ nanosheets, which is almost negligible for the $\mathrm{Mo}_{2} \mathrm{CT}_{x}$ film. The inset of Figure $1 \mathrm{~d}$ is indicative of the Tyndall scattering effect confirming the colloidal nature and the stability of $\mathrm{Mo}_{2} \mathrm{CT}_{x}$ suspensions in water. The V-shape spectra indicate the presence of free carrier absorption, which typically occurs at longer wavelengths for other materials with low free carrier density. ${ }^{[26]}$ However, owing to the higher free carrier concentration of $\mathrm{Mo}_{2} \mathrm{CT}_{x},{ }^{[7]}$ the whole absorption spectra are featured at shorter wavelengths with a minimum (absorption coefficient, $\alpha$, of $57 \mathrm{~cm}^{-1}$ ) at $\approx 250 \mathrm{~nm}$, representing the onset of free carrier absorption. In Figure 1d, the left gray-shaded part denotes the absorption range dominated by the interband transition (IBT) between valence and conduction bands. While at wavelengths between $\approx 230$ and $330 \mathrm{~nm}$, the intraconduction band absorption is stronger than the relatively weak free carrier absorption, ${ }^{[26]}$ which becomes dominant above $\approx 350 \mathrm{~nm}$. The widespread absorption of $\mathrm{Mo}_{2} \mathrm{CT}_{x}$ nanosheets makes it appealing for photodetectors operating in the visible spectral region.

For further investigations, homogeneous MXene thin films $(\approx 2 \mu \mathrm{m}$ thick) were deposited on nylon membranes (filter papers) via vacuum-assisted filtration from MXene suspensions. The corresponding X-ray diffraction (XRD) patterns of films made of intercalated- and exfoliated- $\mathrm{Mo}_{2} \mathrm{CT}_{x}$ are shown in Figure 1e in comparison to XRD of their parent MAX phase $\left(\mathrm{Mo}_{2} \mathrm{Ga}_{2} \mathrm{C}\right.$ powder). The shift in the (002) peak toward lower angles indicates an increase in the $d$-spacing and $c$-lattice parameter compared to their parent MAX phase. ${ }^{[27]}$ The (002) peak for exfoliated- $\mathrm{Mo}_{2} \mathrm{CT}_{x}$, which is partially intercalated with water, ${ }^{[3,28]}$ is observed at $8.9^{\circ}$, but it was shifted to $4.5^{\circ}$ for intercalated$\mathrm{Mo}_{2} \mathrm{CT}_{x}$ upon further intercalation using tetrabutylammonium hydroxide as explained in the Supporting Information. ${ }^{[27]}$ The scanning electron microscopy (SEM) image (Figure 1f) shows that the fabricated $\mathrm{Mo}_{2} \mathrm{CT}_{x}$ thin film (Figure $1 \mathrm{~g}$ ) is composed of few-layer $\mathrm{Mo}_{2} \mathrm{CT}_{x}$ nanosheets stacked together with their surfaces preferentially aligned parallel to the film.

Furthermore, X-ray photoelectron spectroscopy (XPS) was performed on the fabricated MXene thin films to study their chemical compositions and oxidation states. The corresponding high-resolution XPS spectra for Mo $3 \mathrm{~d}$ and $\mathrm{C} 1 \mathrm{~s}$ in $\mathrm{Mo}_{2} \mathrm{CT}_{x}$ are depicted in Figure 1i,j. The Mo $3 d$ core level (Mo $3 d_{5 / 2}-$ Mo $3 \mathrm{~d}_{3 / 2}$ ) of the $\mathrm{Mo}_{2} \mathrm{CT}_{x}$ sample was fitted with four doublets (eight components) with a fixed area ratio equal to $3: 2$ and doublet separation of $3.18 \mathrm{eV}$. The Mo $3 \mathrm{~d}_{5 / 2}$ components were located at 228.2, 229.3, 230.9, and $232.1 \mathrm{eV}$, respectively. The Mo $3 \mathrm{~d}_{5 / 2}$ component centered at $228.2 \mathrm{eV}$ is associated with unterminated Mo bound to $\mathrm{C}$ present in $\mathrm{Mo}_{2} \mathrm{CT}_{x}$ and with traces of $\mathrm{Mo}_{2} \mathrm{Ga}_{2} \mathrm{C}$. ${ }^{[27,29]}$ While the Mo $3 \mathrm{~d}_{5 / 2}$ component centered at $229.3 \mathrm{eV}$ is assigned to surface Mo atoms bound to $\mathrm{C}$ in $\mathrm{Mo}_{2} \mathrm{CT}_{x}$ nanosheets, where Mo is bound to surface termination species such as $\mathrm{O}, \mathrm{OH}$, and/or F. ${ }^{[27,30]}$ Additionally, the Mo $3 \mathrm{~d}_{5 / 2}$ components centered at 230.9 and $232.1 \mathrm{eV}$ are attributed to $\mathrm{Mo}^{5+}$ and $\mathrm{Mo}^{6+}$ oxidized species, respectively, arising from the inevitable surface oxidation during air exposure. ${ }^{[27,31]}$ The detected $\mathrm{Mo}^{6+}$ specie mainly exists in the form of the amorphous $\mathrm{MoO}_{3}$ and possibly in the form of $\mathrm{Mo}_{4} \mathrm{O}_{11}$, which is comprised of $\mathrm{Mo}^{5+}$ besides the $\mathrm{Mo}^{6+}$ specie. ${ }^{[32,33]} \mathrm{Mo}^{5+}$ may also exist in the form of $\mathrm{Mo}_{2} \mathrm{O}_{5},{ }^{[34,35]}$ which is known to be unstable in air, and readily oxidizes to $\mathrm{MoO}_{3}{ }^{[34]}$ Likewise, other possible phases of $\mathrm{Mo}^{5+}$ hydroxyoxides (e.g., $\mathrm{MoO}(\mathrm{OH})_{3}$ and $\mathrm{MoO}_{2}(\mathrm{OH})$ ) are also known to spontaneously oxidize in air forming $\mathrm{Mo}^{6+}$ phases. ${ }^{[36]}$ Accordingly, by the time our devices are measured in air, any possible amount of $\mathrm{Mo}^{5+}$ hydroxyoxides or oxides, other than $\mathrm{Mo}_{4} \mathrm{O}_{11}$, would wane in favor of forming more $\mathrm{Mo}^{6+}$ oxides. Anyway, it is noteworthy that $\mathrm{Mo}^{5+}$-based phases are expected to be very minimal owing to the low content of the $\mathrm{Mo}^{5+}$ specie $(\approx 7 \%)$ within the sampling depth of XPS (Figure 1i). Meanwhile, the Mo bound to $\mathrm{C}$ in $\mathrm{Mo}_{2} \mathrm{CT}_{x}$ remains to be the most abundant species with more than $50 \%$ of the total Mo atoms present in the XPS sampling depth of the MXene film.

The high-resolution spectrum for $\mathrm{C} 1 \mathrm{~s}$ core level of $\mathrm{Mo}_{2} \mathrm{CT}_{x}$ (Figure 1j) was also fitted using seven components located at 283.1, 283.8, 284.4, 284.8, 286.2, 288.1, and $290.4 \mathrm{eV}$ corresponding to $\mathrm{C}-\mathrm{Mo}, \mathrm{C}-\mathrm{Mo}-\mathrm{O}, \mathrm{C}=\mathrm{C}(\mathrm{sp} 2), \mathrm{C}-\mathrm{C} / \mathrm{C}-\mathrm{H}$ (sp3), $\mathrm{C}-\mathrm{O}, \mathrm{C}=\mathrm{O}$, and $(\mathrm{O}-\mathrm{C}=\mathrm{O}$ and $\mathrm{C}-\mathrm{F})$ bonds, respectively. ${ }^{[27]}$ 
a

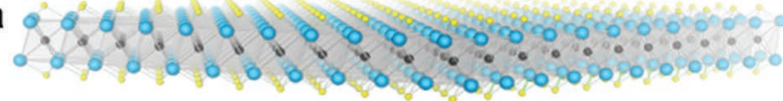

- Mo $\bullet \mathrm{C} \odot$ Surface moieties, $T_{x}(\mathrm{O}, \mathrm{F}, \mathrm{OH})$
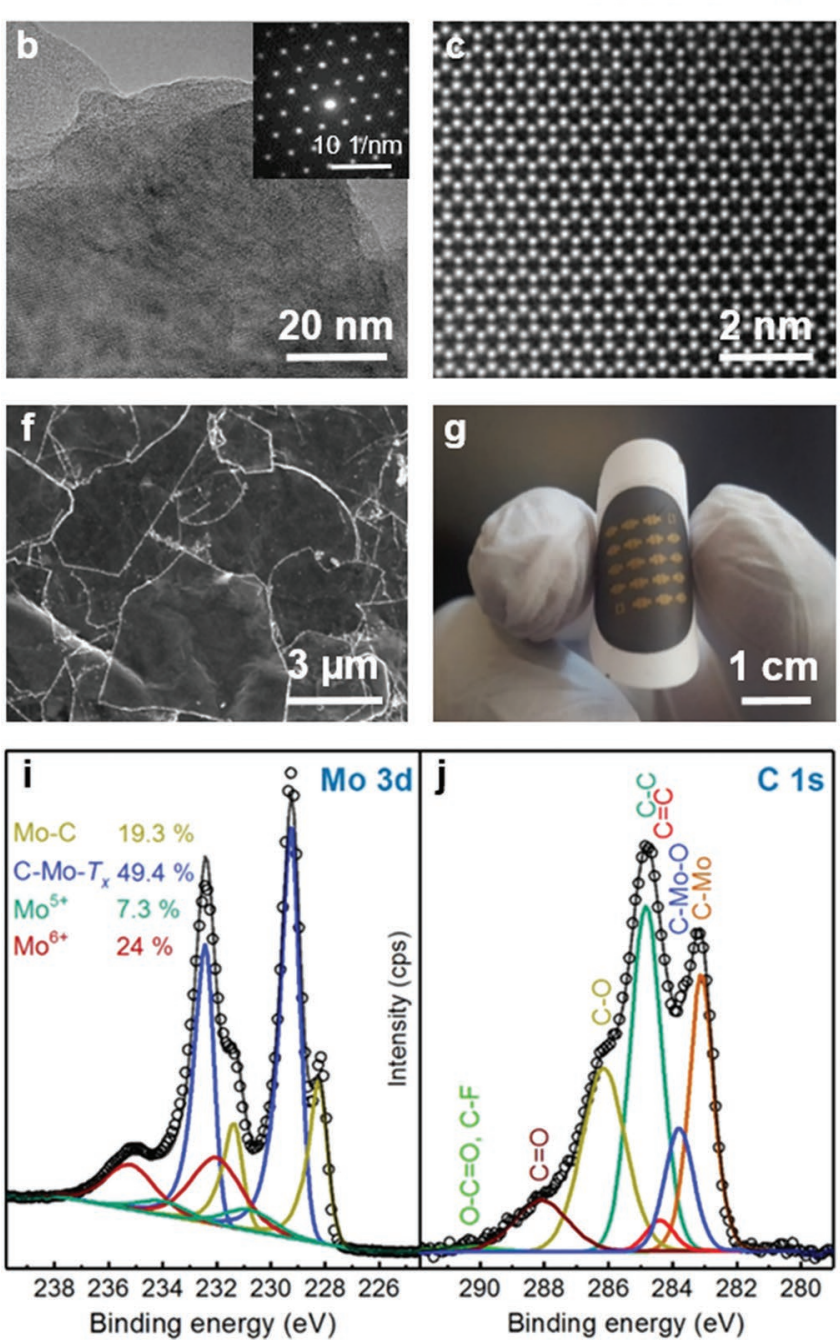
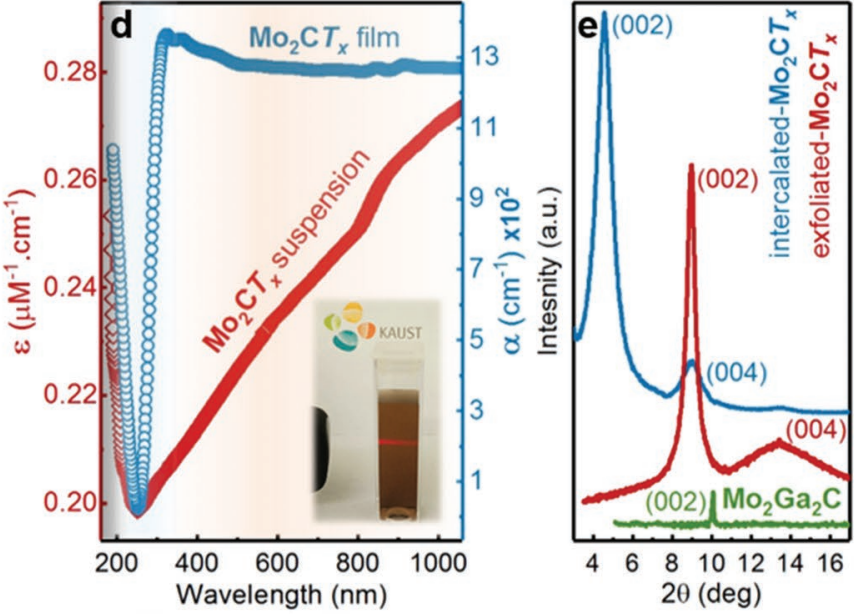

h
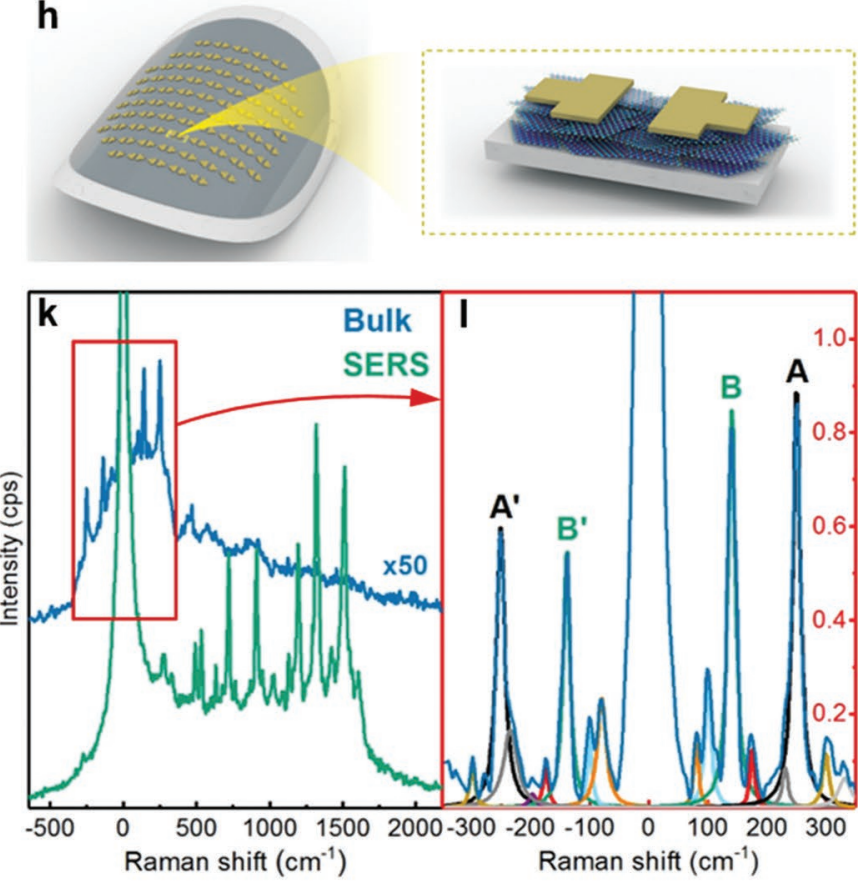

Figure 1. a) Schematic illustration of a delaminated $\mathrm{Mo}_{2} \mathrm{CT}_{x}$ nanosheet: One layer of carbon is sandwiched between two layers of Molybdenum and the surface is randomly terminated with functional surface moieties, $\mathrm{T}_{x}$ : $(-\mathrm{OH},-\mathrm{O} \text {, and }-\mathrm{F})_{x}$. b) $\mathrm{TEM}_{\mathrm{image}}$ of few-layered $\mathrm{Mo}_{2} \mathrm{CT}_{x}$ nanosheets. Inset: Fast Fourier transform (FFT) pattern. c) High-resolution STEM image of $\mathrm{Mo}_{2} \mathrm{CT}_{x}$. Note the hexagonal structure of the basal planes and high crystallinity of the $\mathrm{Mo}_{2} \mathrm{CT}_{x}$ nanosheet with no obvious nanometer-scale defects. d) UV-vis-NIR absorption spectra of $\mathrm{Mo}_{2} \mathrm{CT}_{x}$ film (blue, $1.8 \mu \mathrm{m}$ ) and dispersed in water (red, $0.6 \mathrm{mg} \mathrm{mL}^{-1}$ ) with a minimum at $\approx 250 \mathrm{~nm}$, representing the onset of free carrier absorption. Inset: A photograph showing the Tyndall scattering effect in stable colloidal water-suspension of $\mathrm{Mo}_{2} \mathrm{CT}_{x}$ nanosheets. e) XRD patterns (vertically displaced for $\mathrm{Clarity}$ ) of $\mathrm{Mo}_{2} \mathrm{Ga}_{2} \mathrm{C}$ (green), exfoliated-Mo $\mathrm{O}_{2} \mathrm{CT}_{x}$ (red), and intercalated- $\mathrm{Mo}_{2} \mathrm{CT}_{x}$ (blue) at low Bragg's angles. f) SEM micrograph showing the surface morphology of a Mo $\mathrm{CT}_{x}$ thin film. g) Photograph and h) schematic illustration of an array of two-terminal, parallel-type thin film $\mathrm{Mo}_{2} \mathrm{CT}_{x}$-based flexible photodetectors. i) High-resolution XPS spectrum of the Mo $3 \mathrm{~d}$ and j) $\mathrm{C} 1 \mathrm{~s}$ core levels. k) Micro-Raman spectroscopy of $\mathrm{Mo}_{2} \mathrm{CT}_{x}$ at $633 \mathrm{~nm}$. (Blue) Resonant Raman scattering spectrum of Mo $\mathrm{CT}_{x}$ (Bulk, obtained by averaging more than 1000 spectra). (Green) A surface-enhanced Raman scattering (SERS) spectrum, displayed for comparison, obtained at one position on the Au electrodes. I) Low-frequency range of the Raman spectrum of the bare $\mathrm{Mo}_{2} \mathrm{CT}_{x}$ film. The spectrum is analyzed by Lorentzian functions after subtraction of the broad baseline.

Figure S7 in the Supporting Information displays the highresolution XPS spectra of $\mathrm{F} 1 \mathrm{~s}$ and $\mathrm{O}$ 1s regions of the $\mathrm{Mo}_{2} \mathrm{CT}_{x}$ film. The corresponding high-resolution XPS spectra of the other studied MXenes $\left(\mathrm{Ti}_{3} \mathrm{C}_{2} \mathrm{~T}_{x}, \mathrm{Nb}_{2} \mathrm{CT}_{x}, \mathrm{~T}_{2} \mathrm{CT}_{x}\right.$, and $\left.\mathrm{V}_{2} \mathrm{CT}_{x}\right)$ are displayed in Figures S8-S11 in the Supporting Information.

Next, e-beam evaporation was used to deposit gold electrodes $\left(\approx 50 \mathrm{~nm}\right.$ thick) on the fabricated $\mathrm{Mo}_{2} \mathrm{CT}_{x}$ films, giving rise to mechanically flexible arrays of two-terminal, parallel-type devices with a channel length and width of $70 \mu \mathrm{m}$ and $1 \mathrm{~mm}$, respectively (see Figure $1 \mathrm{~g}, \mathrm{~h}$ ). Thereupon, these $\mathrm{Mo}_{2} \mathrm{CT}_{x}$ films were characterized by micro-Raman spectroscopy imaging in large areas across the two electrodes of single devices. The corresponding spectral analysis (Figure 1k,l) unveiled the lowfrequency fingerprint spectrum of our $\mathrm{Mo}_{2} \mathrm{CT}_{x}$ MXene and 
elucidated a significant heterogeneity of its surface chemistry. By exploiting the surface-enhanced Raman scattering (SERS) effect, triggered by the Au electrodes, we were able to measure signals arising from nanoscale volumes defined by nanogaps and confined on the outer most surface of the nanosheets. The measured signals have evidenced a very high spectral variability, at submicron scale level, in the high-frequency range, i.e., above $600 \mathrm{~cm}^{-1}$. In contrast, bare $\mathrm{Mo}_{2} \mathrm{CT}_{x}$ scattering results, though of very small intensity, were spatially quite uniform. Thus, by averaging hundreds of consecutive spectra, it was possible to identify the main molecular vibrational modes within the $\mathrm{Mo}_{2} \mathrm{CT}_{x}$ network including shear and interlayer breathing modes. The Lorentzian shape analysis of the Stokes (S) and anti-Stokes (AS) sides of the spectrum (Figure 11) in the $(-500,500) \mathrm{cm}^{-1}$ range allowed for the identification of up to ten vibrations with high accuracy. The assignment of the identified peaks is summarized in Table S1 in the Supporting Information, and detailed in the Supporting Information. We have also highlighted a photon-electron-phonon anisotropic resonance process relying on the observed anomalously low S/AS intensity ratios: $\approx 1.3$. Interestingly, such a value is rather far from what can be estimated $(\approx 10)$ for considered peak couples, $\Delta v_{\text {S-AS }}=500 \mathrm{~cm}^{-1}$, following the Bose-Einstein statistics, generally used to describe thermally activated phononic excitations. For more details, refer to Figures S12-S14 in the Supporting Information and the corresponding Raman structural characterization.

Next, to study the broadband visible photoresponse of MXene thin films, we measured their spectral sensitivity as a function of wavelength. In particular, we have examined $\mathrm{Mo}_{2} \mathrm{CT}_{x}$ thin films in the $400-800 \mathrm{~nm}$ range focusing the tunable radiation of a supercontinuum white laser excitation on a spot size of $\leq 25 \mu \mathrm{m}$ at the center between the electrodes (70 $\mu \mathrm{m}$ apart), partially on one electrode and on a large area covering the whole device by simply defocusing the illumination (Figure 2a; Figure S15, Supporting Information). The spectral profile of thin films made of $\mathrm{Mo}_{2} \mathrm{CT}_{x}$ demonstrated a spectral response in the range of 500 to $800 \mathrm{~nm}$ (Figure 2a; Figure S15b, Supporting Information), highlighting the resonant response of the device in the visible region. This spectral response lies within the window of free carrier absorption as illustrated in the measured UV-vis-NIR absorption spectrum of $\mathrm{Mo}_{2} \mathrm{CT}_{x}$ (Figure 1d). Furthermore, we performed a fixed bias $(0.5 \mathrm{~V})$ micro-photocurrent imaging as shown in Figure S16 in the Supporting Information. The corresponding micro-photocurrent map, obtained at a representative wavelength of $633 \mathrm{~nm}$, is in agreement with the spectral sensitivity of $\mathrm{Mo}_{2} \mathrm{CT}_{x}$ devices. It shows a rather homogeneous signal
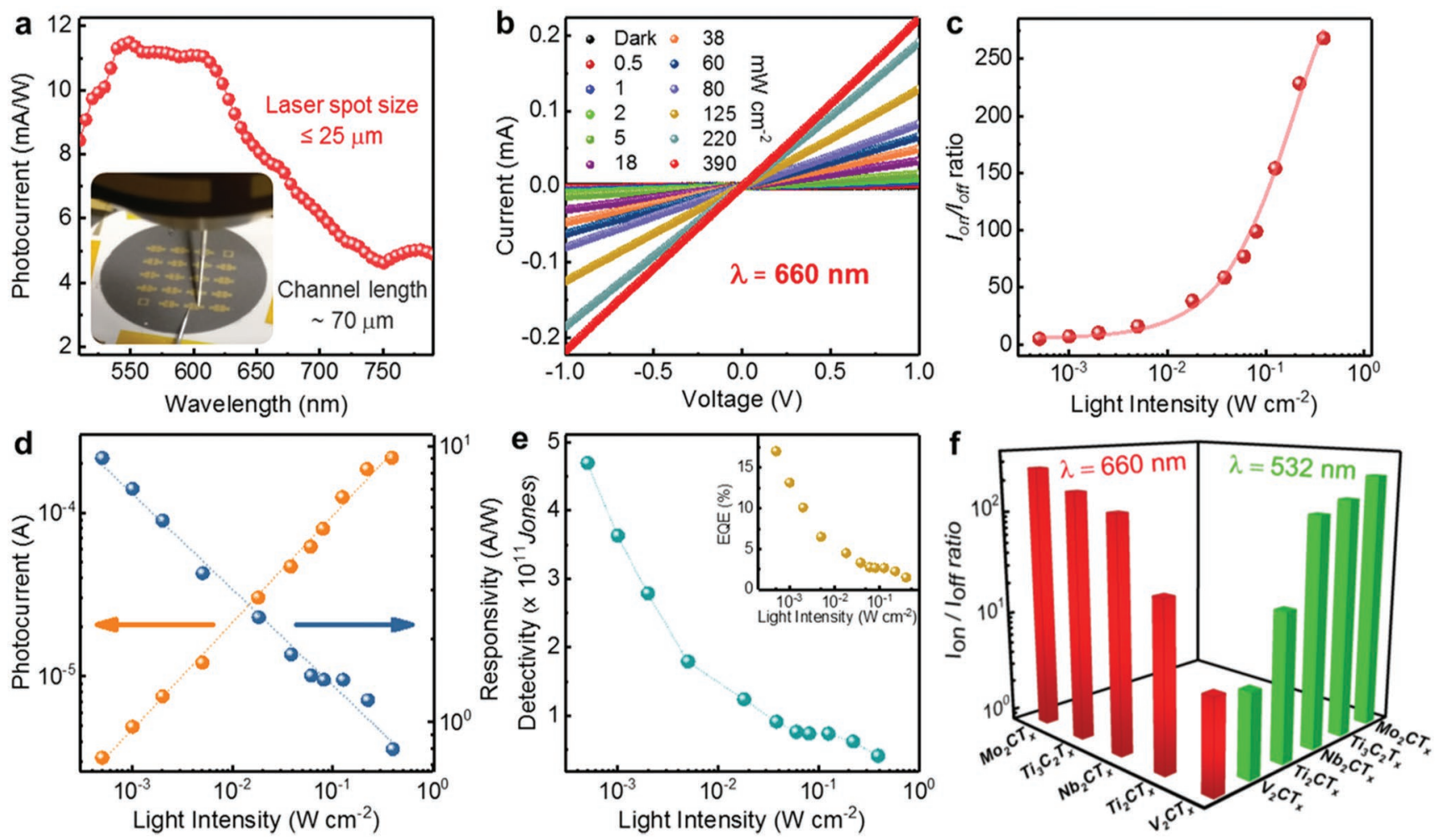

Figure 2. a) Photoresponse of a $\mathrm{Mo}_{2} \mathrm{CT}_{x}$ thin film photodetector as a function of wavelength, under $0.7 \mathrm{~V}$ bias voltage. Inset: Photograph of the photocurrent setup showing one of the investigated samples, illumination objective, and the contact electrodes. The spot size of the supercontinuum white laser excitation was $\leq 25 \mu \mathrm{m}$. b) Linear scale current-voltage $(I-V)$ characteristics of the $\mathrm{Mo}_{2} \mathrm{CT}_{x}$ thin film photodetector in the dark and under different light intensities at a wavelength of $660 \mathrm{~nm}$ with a bias voltage of $\pm 1 \mathrm{~V}$. c) Ratios of the photocurrent to the dark current of the $\mathrm{Mo}_{2} \mathrm{CT}_{x}$ thin film photodetector as function of different light intensities. d) (Left) Photocurrents of the $\mathrm{Mo}_{2} \mathrm{CT}_{x}$ thin film photodetector as a function of different light intensities with respect to the dark state of the device. (Right) Responsivity of the photodetector as a function of different light intensities. e) The detectivity and EQE (inset) of the photodetector as a function of different light intensities. f) Ratios of the photocurrent of different MXene photodetectors with respect to the dark current, measured at wavelengths of $660 \mathrm{~nm}$ (red bars) and $532 \mathrm{~nm}$ (green bars). All the values were obtained at light intensities of 0.39 and $0.41 \mathrm{~W} \mathrm{~cm}^{-2}$ for wavelengths of 660 and $532 \mathrm{~nm}$, respectively. Results shown in (c-e) are obtained considering an excitation at $660 \mathrm{~nm}$. 
superimposed on a bias-dependent current ground level (refer to the Supporting Information for more details). On a side note, it has not escaped our notice to consider any possible photoabsorption by the oxidized Mo species (see Figure 1i). However, such Mo oxidized species (e.g., $\mathrm{MoO}_{3}$ and/or $\mathrm{Mo}_{4} \mathrm{O}_{11}$ ) are known to have wide a band gap of $\approx 3.2 \mathrm{eV}$ or even larger, ${ }^{[32,37-41]}$ which rules out the possibility of their contribution in the visible photoresponse of our $\mathrm{Mo}_{2} \mathrm{CT}_{x}$ thin films.

Following the spectral response depicted in Figure 2a, we examined the visible light detection of $\mathrm{Mo}_{2} \mathrm{CT}_{x}$ thin films, under ambient conditions, at a representative wavelength of $660 \mathrm{~nm}$ with various light intensities. The linear and symmetrical behavior of the $I-V$ curves of the $\mathrm{Mo}_{2} \mathrm{CT}_{x}$ (Figure $2 \mathrm{~b}$ ) indicates an Ohmic contact has formed between the thin film and $\mathrm{Au}$ electrodes. The current gradually increases with the light intensity, as a result of the incremented number of excited carriers, yielding a maximum $I_{\text {on }} / I_{\text {off }}$ ratio of $\approx 2 \times 10^{2}$ at a light intensity and bias voltage of $0.39 \mathrm{~W} \mathrm{~cm}^{-2}$ and $1 \mathrm{~V}$, respectively, as shown in Figure 2c. The $I_{\text {on }} / I_{\text {off }}$ ratio for the $\mathrm{Mo}_{2} \mathrm{CT}_{x}$ thin film as a function of film thickness demonstrates a saturation effect indicating a photocurrent level almost independent of the film thickness in the range from 1.5 to $4.0 \mu \mathrm{m}$ (Figure S17, Supporting Information), that characterizes the full coverage of the substrate. The photocurrent is obtained at different incident light intensities by subtracting the dark current from the current recorded under illumination (Figure 2d). The photocurrent strongly depends on the incident light intensities and increases steeply from $1 \times 10^{-6}$ to $2 \times 10^{-4} \mathrm{~A}$ as the light intensity increases from $5 \times 10^{-4}$ to $0.39 \mathrm{~W} \mathrm{~cm}^{-2}$.

To evaluate the performance of the $\mathrm{Mo}_{2} \mathrm{CT}_{x}$ photodetectors, their responsivity $(R)$, specific detectivity $\left(D^{*}\right)$, and external quantum efficiency (EQE) were calculated under incident light of $660 \mathrm{~nm}$ and a bias voltage of $-1 \mathrm{~V}$ and are presented in Figure 2 d,e. $R, D^{*}$, and EQE are calculated based on previous reports with the assumption that the dark current is primarily dominated by the shot noise (Table S2, Supporting Information). ${ }^{[42-44]}$ At light intensities as low as $5 \times 10^{-4} \mathrm{~W} \mathrm{~cm}^{-2}$, our device reaches a responsivity of $9 \mathrm{~A} \mathrm{~W}^{-1}$ and decreases with increasing light intensity (Figure 2d). The drop in responsivity is attributed to the filling of the trap states present in the $\mathrm{Mo}_{2} \mathrm{CT}_{x}$ thin film that might be originated from the defects and/ or surface-terminated functional groups. Besides, increasing the illumination at high level enhances the recombination of generated hot carriers while progressively reduces the density of available states in the conduction band, resulting in a saturation of the photocurrent and a gradual decrease in the $R{ }^{[45]}$ The calculated maximum $D^{*}$ and EQE, under illumination of $660 \mathrm{~nm}$, are $4.7 \times 10^{11}$ Jones and 17 , respectively, and they gradually decrease with increasing light intensity (Figure 2d). In addition, our solution-processed $\mathrm{Mo}_{2} \mathrm{CT}_{x}$ photodetector arrays demonstrate very high cell-to-cell and batch-to-batch reliabilities (Figure S18, Supporting Information).

We also examined the photodetection performance of $\mathrm{Mo}_{2} \mathrm{CT}_{x}$ at another visible wavelength of $532 \mathrm{~nm}$ (Figure S19, Supporting Information), and it exhibited similar performance to that obtained at $660 \mathrm{~nm}$, which correlates with the broad visible band selectivity shown in Figure 2a. The visible-light photoresponse of other MXene compositions was also studied and the results are summarized in Figure 2f, and Figures S20 and S21 in the Supporting Information. All of the photodetectors responded to the 660 and $532 \mathrm{~nm}$ light with little variation in the detection performance, which depends on the characteristic optical absorption, electrical conductivity, and free carrier concentration of the individual MXenes.

In view of the broadband absorption of $\mathrm{Mo}_{2} \mathrm{CT}_{x}$ (Figure $1 \mathrm{~d}$ ), we also examined our devices under UV (325 nm) and NIR (1064 nm) illumination, near both edges of the visible band. Interestingly, despite being within the free carrier absorption regime as indicated by the UV-vis-NIR absorption spectra in Figure $1 \mathrm{~d}$, very weak photoresponse was obtained at both spectral ranges (Figure S22, Supporting Information). We attribute this to the presence of characteristic surface plasmons (SPs), collective oscillations of free carriers, supported by $\mathrm{Mo}_{2} \mathrm{CT}_{x}$ nanosheets. In principle, the possibility to excite an SP resonating at the same frequency of incident light is known to enhance the device performance of many applications and is the distinctive characteristic of a plasmonic device. Hence, we believe that our devices have lower performances at nonvisible wavelengths because they do not match any of the resonance frequencies of the SPs that are potentially supported by the $\mathrm{Mo}_{2} \mathrm{CT}_{x}$ nanosheets. A thorough explanation for our hypothesis is provided in the Supporting Information, where we discuss the physical electromechanical properties of a multilayer $\mathrm{Mo}_{2} \mathrm{CT}_{x}$ cluster in comparison with gold as a typical example for conventional plasmonic materials.

To validate our hypothesis, we implemented a combination of STEM and ultrahigh resolution EELS to investigate the presence of SPs supported by multilayer $\mathrm{Mo}_{2} \mathrm{CT}_{x}$ nanosheets. Figure $3 \mathrm{a}$ displays a typical low-loss EEL spectra (with zero-loss-peak (ZLP) subtracted) of $\mathrm{Mo}_{2} \mathrm{CT}_{x}$ with four distinct features, identified at $0.3,0.39,2.45$, and $3.42 \mathrm{eV}$. The obtained EELS intensity distribution maps at each feature are shown in the inset of Figure $3 \mathrm{a}$ along with the annular dark field (ADF) STEM micrograph of a truncated triangular $\mathrm{Mo}_{2} \mathrm{CT}_{x}$ nanosheet (58 nm thick). We interpret the peaks at 0.3 and $0.39 \mathrm{eV}$ as the dipole and quadrupole longitudinal modes, respectively, of the SP supported by the $\mathrm{Mo}_{2} \mathrm{CT}_{x}$ nanosheet. Analogous distinct multipolar modes have also been reported for $\mathrm{Ti}_{3} \mathrm{C}_{2} \mathrm{~T}_{x}$ nanosheets. ${ }^{[19]}$ The peak at $2.45 \mathrm{eV}$, distinguished with its homogeneous distribution, is assigned to the transversal SP mode, which is sizeindependent and solely related to the free electron concentration. Further discussion about the differences between longitudinal and transversal SP modes is provided in the STEM/EELS characterization section in the Supporting Information. The EELS absorption edge with an onset at $3.42 \mathrm{eV}$ is ascribed to the interconduction band transitions as well as the IBT extending up to $7 \mathrm{eV}$. EELS maps of SPs for other $\mathrm{Mo}_{2} \mathrm{CT}_{x}$ and $\mathrm{Ti}_{3} \mathrm{C}_{2} \mathrm{~T}_{x}$ nanosheets are depicted in Figures S23-S28 in the Supporting Information.

In principle, our EELS results suggest that the $\mathrm{Mo}_{2} \mathrm{CT}_{x}$ devices would only work at wavelengths in resonance with the supported SPs, be it longitudinal or transversal modes. However, owing to the low resonance energy levels (mid-IR range) of the longitudinal modes, which are beyond the capability of our photoexcitation sources, we directed our attention to the transversal SP mode (refer to the Supporting Information for more details about the nature of the longitudinal and transversal SP modes). The energy window of the transversal 

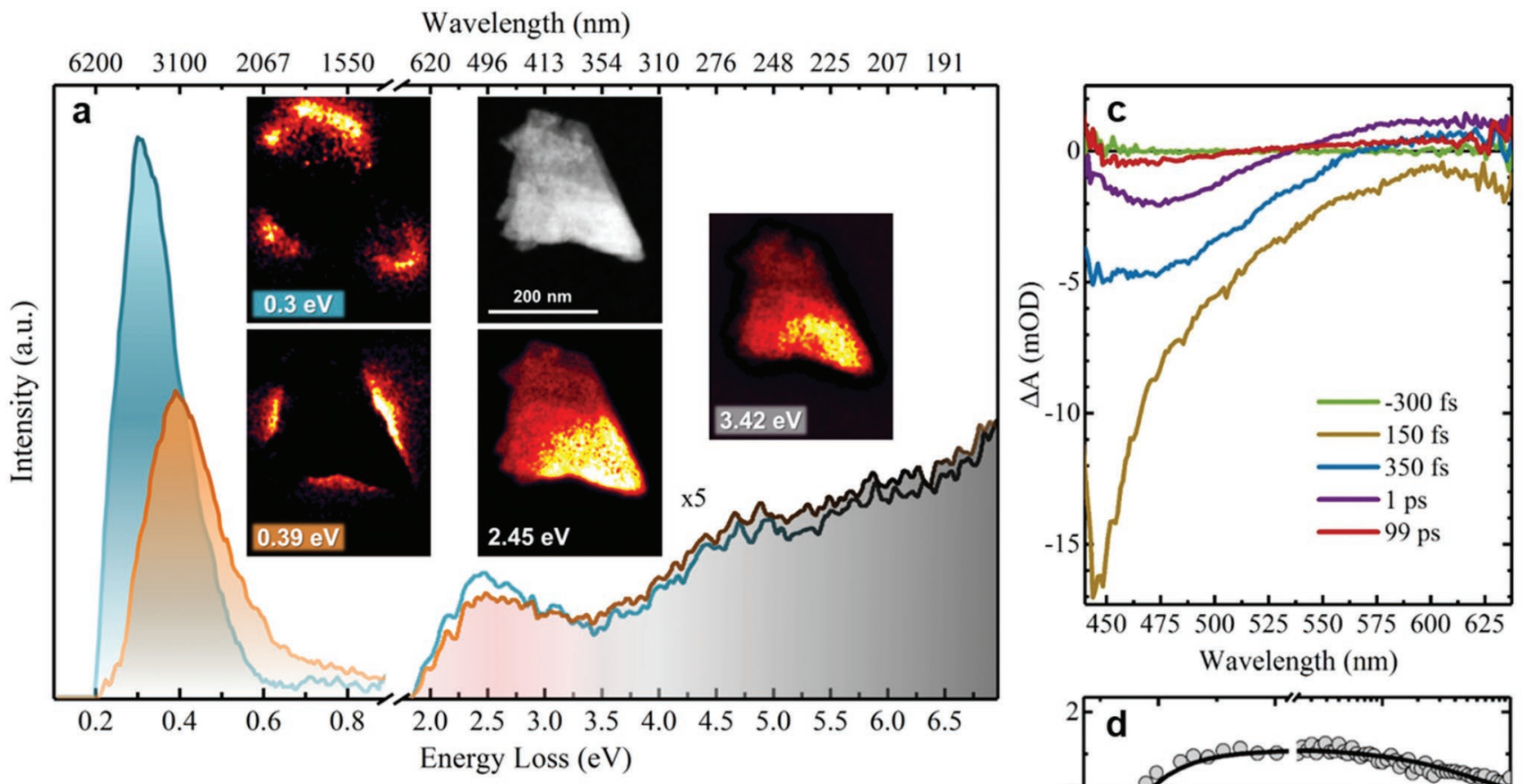

b

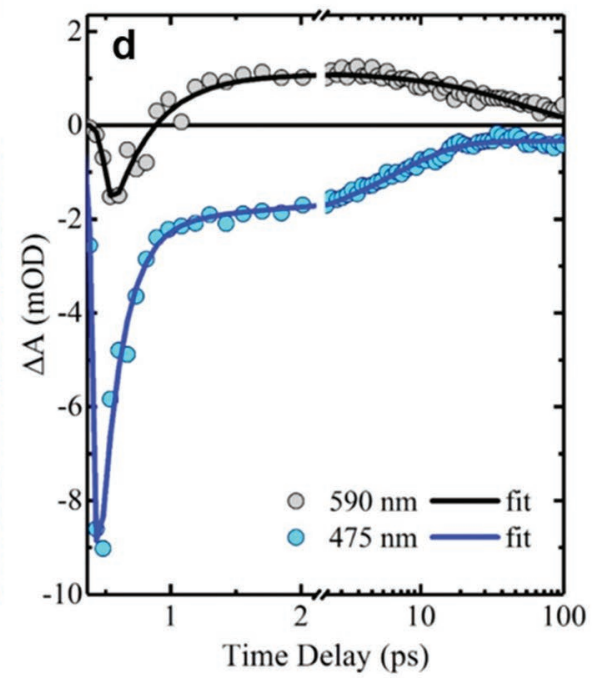

Figure 3. a) ZLP-subtracted EELS acquired on a truncated triangular nanosheet of $\mathrm{Mo}_{2} \mathrm{CT}_{x}(\approx 58 \mathrm{~nm} \pm 1$ at the center). The spectra are normalized to the transversal SP peak at $2.45 \mathrm{eV}$ (highlighted in red) and magnified by a factor of 5 above $1.7 \mathrm{eV}$. Insets: $\mathrm{ADF}^{-\mathrm{STEM}}$ micrograph of the $\mathrm{Mo}_{2} \mathrm{CT}_{x}$ nanosheet on a $\mathrm{Si}_{3} \mathrm{~N}_{4}$ supporting membrane (black area), and the EELS fitted intensity maps of the corresponding longitudinal SP modes; namely, dipole $(0.3 \mathrm{eV})$ and quadrupole $(0.39 \mathrm{eV})$, in addition to the transversal SP mode $(2.45 \mathrm{eV})$ and the IBT (starting at $3.42 \mathrm{eV})$. b) Schematic representation of the photodetector under illumination showing the migration process of the plasmon-assisted hot electrons toward the biased gold electrodes. c) Time-resolved visible transient absorption spectra of $\mathrm{Mo}_{2} \mathrm{CT}_{x}$ at different time slots. d) Kinetic traces of the $\mathrm{GSB}$ recovery and ESA decay of $\mathrm{Mo}_{2} \mathrm{CT}_{x}$ extracted at 475 and $590 \mathrm{~nm}$, respectively. These data were collected under excitation wavelength of $330 \mathrm{~nm}$.

SP mode is highlighted in red in Figure 3a, and in the ellipsometric results obtained for $\mathrm{Mo}_{2} \mathrm{CT}_{x}$ thin films (see Figure S29 and Table S3, Supporting Information). Once the photoactive $\mathrm{Mo}_{2} \mathrm{CT}_{x}$ thin film is illuminated at wavelengths with matching resonance, plasmon-assisted hot carriers are generated and efficiently separated to the Au electrodes maintained under an applied electric field. We believe that the photocurrent generation in $\mathrm{Mo}_{2} \mathrm{CT}_{x}$ is principally controlled by plasmon-assisted hot electrons, with marginal photo-thermoelectric and bolometric effects (Figure S30, Supporting Information). ${ }^{[7,27]}$ The schematic representation in Figure $3 \mathrm{~b}$ illustrates the migration process of the plasmon-assisted hot carriers toward the biased gold electrodes. These results not only correlate very well with our hypothesis but are also considered as the first report evidencing SPs in MXenes other than $\mathrm{Ti}_{3} \mathrm{C}_{2} \mathrm{~T}_{x}$.

To elucidate the dynamics of the generated plasmonassisted hot carriers, i.e., electrons, upon excitation, we conducted femtosecond visible transient absorption measurements for our MXene nanosheets. Figure 3c shows the transient spectra of $\mathrm{Mo}_{2} \mathrm{CT}_{x}$ nanosheets obtained under UV-laser excitation of $330 \mathrm{~nm}$. The transient absorption spectra are characterized by a strong ground state bleach (GSB), maximized at $\approx 450 \mathrm{~nm}$ at early times. Generally, in $2 \mathrm{D}$ sheets, the plasmon peaks are expected to be broad and featureless due to high damping effect form electron-phonon interactions. ${ }^{[46,47]}$ In our measurement, the GSB underwent an ultrafast process 
within $\approx 200 \mathrm{fs}$, in which the GSB diminished, and simultaneously a positive feature, excited state absorption (ESA), centralized at $\approx 590 \mathrm{~nm}$, evolved. Such an ultrafast femtosecond process corroborates the existence of SPs as evidenced by EELS. Subsequently, both GSB and ESA recovered into zero with time scales of $\approx 13$ and $53 \mathrm{ps}$, respectively. The lifetimes were extracted by fitting the kinetic traces at 475 and $590 \mathrm{~nm}$ as shown in Figure 3d. The observed dynamics are attributed to the following processes: 1) After photon excitation, SPs were directly populated within $120 \mathrm{fs}$ (temporal resolution of our technique), as reflected by the strong GSB at early times. ${ }^{[48]}$ 2) According to Landau damping model, the plasmon can transfer its energy to the formation of an electron-hole pair, with a time scale of $\approx 100 \mathrm{fs}$, for metallic materials. ${ }^{[48-50]} \mathrm{How}$ ever, in our case, this process took about 200 fs to occur, in which the formation of ESA is attributed to the electron features, while the strong quenching of GSB is ascribed to the hole features. 3) Following the electron-hole formation, the recombination process for the electron was $\approx 53 \mathrm{ps}$, and $\approx 13 \mathrm{ps}$ for the hole. This variation in lifetimes can be attributed to the presence of active hole-trapping sites that are filled afterward by the populated electrons within $53 \mathrm{ps}$. The relatively slow recombination dynamics compared to classical plasmonic metals can be attributed to the absence of metallic structures as previously reported. ${ }^{46]}$ Such slow relaxation is indicative of scattering with phonons and few other electrons, which correlates well with unexpected S/AS intensity ratios observed in the low-frequency Raman. This finding concurs with the relatively low free carrier density $\approx 10^{20} \mathrm{~cm}^{-3}$, ,7] which is $\approx 4$ orders lower than metals. To the best of our knowledge, this is the first report for ultrafast visible transient absorption of any MXene. We also obtained the kinetic traces of $\mathrm{Ti}_{3} \mathrm{C}_{2} \mathrm{~T}_{x}$ along with their extracted lifetimes (Figure S31, Supporting Information).

Next, we investigated the dynamic performance of $\mathrm{Mo}_{2} \mathrm{CT}_{x}$ photodetector by measuring their response time for the rise and decay of the photocurrent upon switching the light ON and OFF. Figure 4a shows the photocurrent of the photodetector under alternating $\mathrm{ON}$ and $\mathrm{OFF}$ cycles at a wavelength of $660 \mathrm{~nm}$ with a light intensity of $0.22 \mathrm{~W} \mathrm{~cm}^{-2}$. The photocurrent of the $\mathrm{Mo}_{2} \mathrm{CT}_{x}$ thin films sharply rose and decayed upon multiple switching cycles at the millisecond level, demonstrating good reliability and reversibility of the photodetector. To check the stability of the photodetectors, the photocurrent was measured under continuous illumination of $660 \mathrm{~nm}$ light in ambient conditions, as shown in Figure 4b. The device maintained over $80 \%$ of the initial photocurrent even after $12000 \mathrm{~s}$. In addition, our air-exposed $\mathrm{Mo}_{2} \mathrm{CT}_{x}$ thin film channel exhibits weak charge transfer between $\mathrm{Mo}_{2} \mathrm{CT}_{x}$ and the absorbed $\mathrm{O}_{2}$ or $\mathrm{H}_{2} \mathrm{O}$ molecules, such that only less excited carriers (hot electrons) contribute to the charge transfer, and thus a stable photodetection operation was achieved. ${ }^{[51,52]}$ In order to verify the charge-transfer effect, we measured the dark current of the $\mathrm{Mo}_{2} \mathrm{CT}_{x}$ in air and in vacuum. The dark current measured in air for $\mathrm{Mo}_{2} \mathrm{CT}_{x}$ films was not different from that measured under vacuum (Figure S32, Supporting Information), suggesting that the devices have no significant interaction with the ambient $\mathrm{H}_{2} \mathrm{O}$ or $\mathrm{O}_{2}$ molecules. Besides, the high photostability of our $\mathrm{Mo}_{2} \mathrm{CT}_{x}$ nanosheets can be ascribed to their high crystal quality and few nanometer-scale defects (Figure 1b,c), which benefits the structural and chemical integrity of the device. On the other hand, the stability of the $\mathrm{Ti}_{3} \mathrm{C}_{2} \mathrm{~T}_{x}$ MXene thin film is strongly deteriorated with increasing illumination time (Figure S33, Supporting Information). The spontaneous partial oxidation of $\mathrm{Ti}_{3} \mathrm{C}_{2} \mathrm{~T}_{x}$ MXene ${ }^{[14]}$ leads to the formation of titanium oxides (e.g., $\mathrm{TiO}_{2}$ ), on which $\mathrm{O}_{2}$ molecules are generally known to be adsorbed. ${ }^{[53]}$ In consequence, the air-exposed $\mathrm{Ti}_{3} \mathrm{C}_{2} \mathrm{~T}_{x}-\mathrm{TiO}_{2}$ hybrid channel exhibits strong charge-transfer effect between $\mathrm{TiO}_{2}$ and the adsorbed $\mathrm{O}_{2}$, allowing for the contribution of more photoinduced carriers in the charge transfer process, and thus decreasing the stability. This analogy was further confirmed by obtaining the dark current of $\mathrm{Ti}_{3} \mathrm{C}_{2} \mathrm{~T}_{x}$ films in vacuum and in air. The dark current measured in vacuum was significantly higher than that measured in air, confirming the strong charge-transfer effect between $\mathrm{TiO}_{2}$ and $\mathrm{O}_{2}$ molecules.

Meanwhile, the stability of the fabricated photodetectors was checked as a function of storage time, where no obvious deterioration has been observed. The $I_{\mathrm{on}} / I_{\text {off }}$ ratio remained almost the same over the course of more than 30 days of storage inside a vacuum desiccator (Figure $4 \mathrm{c}$ ). These results suggest that the $\mathrm{Mo}_{2} \mathrm{CT}_{x}$ thin film devices are stable under vacuum storage conditions, highlighting their stable and durable photodetection operation. However, their stability in oxygen-containing environments remains to be properly investigated. Moreover, knowing that MXenes have demonstrated excellent mechanical flexibility, ${ }^{[54]}$ we investigated the photodetection properties of $\mathrm{Mo}_{2} \mathrm{CT}_{x}$ thin films under various mechanical deformations (different bending radii). The initial $I_{\text {on }} / I_{\text {off }}$ ratio values, obtained at $660 \mathrm{~nm}$, as a function of the bending radius, remained unaltered even at a bending radius of $\approx 3 \mathrm{~mm}$ (see Figure $4 \mathrm{~d}$ ). A similar performance was also obtained after 500 bending cycles in the outward direction with a bending radius of $\approx 3 \mathrm{~mm}$, without any significant deterioration in the performance (Figure $4 \mathrm{e}$ ). The arrays of $\mathrm{Mo}_{2} \mathrm{CT}_{x}$ thin film photodetectors, fabricated on nylon membranes, are readily bendable under multiple and repeated deformations. This illustrates their potential for harsh optoelectronics against mechanical stress and other flexible applications.

In summary, we have fabricated flexible MXene thin film photodetectors operating in the visible spectral region. Their photoresponse is dominated by the intrinsic plasmon-assisted hot carrier generation without the need for integration with other metallic plasmonic structures, as previously demonstrated with several 2D materials. ${ }^{[55]}$ Despite being the first demonstration of plasmonic photodetection in MXenes, the performance of $\mathrm{Mo}_{2} \mathrm{CT}_{x}$ thin films considering their $R$ and $D^{*}$ is surpassing the majority of previously reported visible-band photodetectors based on solution-processed 2D materials. A comparison of several literature reports is summarized in Table S4 in the Supporting Information. In addition to their attractive performance and solution processability, we have revealed that our MXene-based devices possess additional set of advantages including full visible spectrum coverage, highly stable operation, and mechanical flexibility. On the top of that, by means of high-resolution STEM-EELS, we have been able, for the first time, to image the spatial and energy distribution of SP modes over individual $\mathrm{Mo}_{2} \mathrm{CT}_{x}$ nanosheets. Further, using 

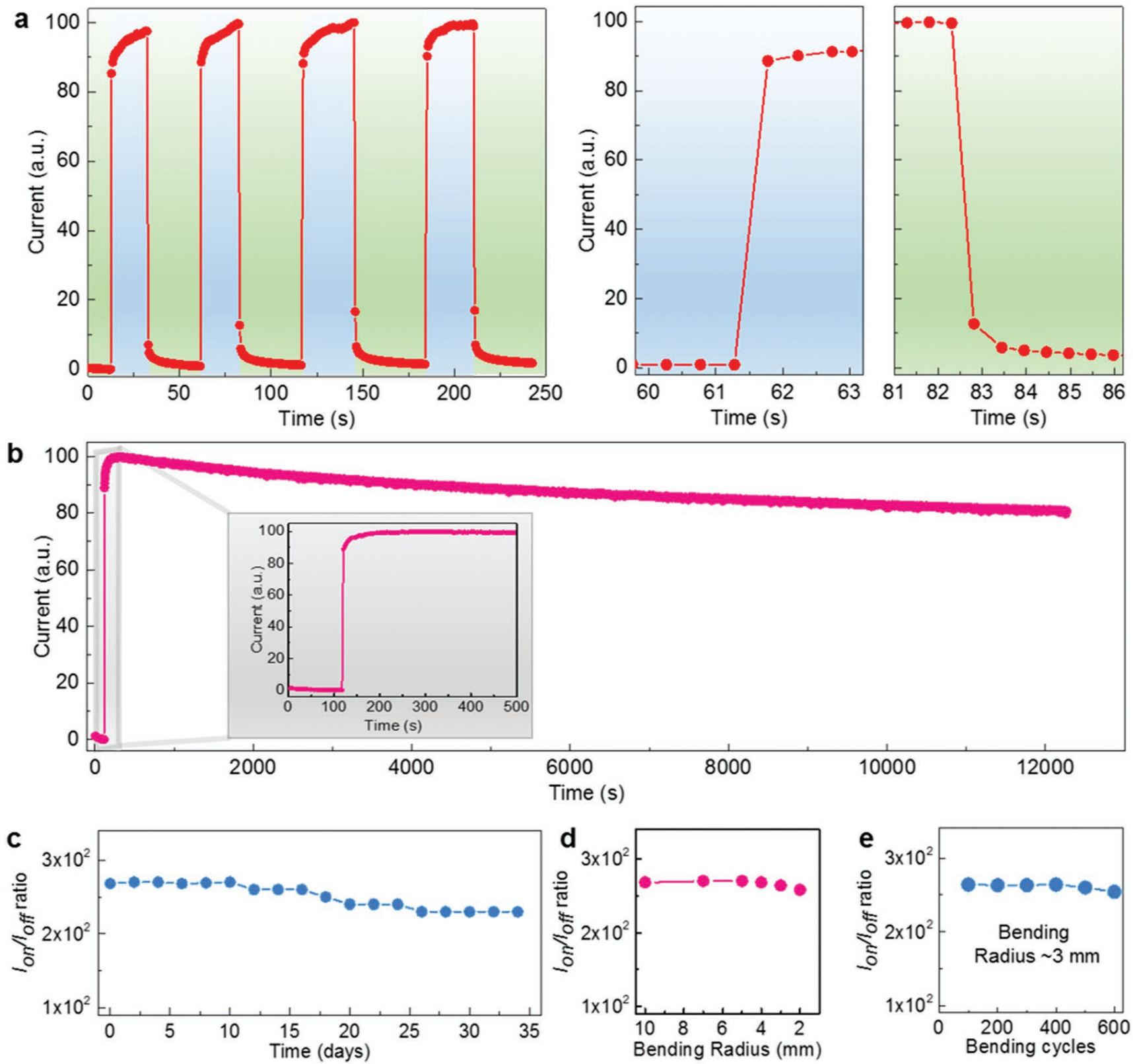

Figure 4. a) Photoresponse behavior of $\mathrm{Mo}_{2} \mathrm{CT}_{x}$ thin film photodetector under alternating $\mathrm{ON}$ and OFF cycles at a wavelength of $660 \mathrm{~nm}$ with a light intensity of $0.22 \mathrm{~W} \mathrm{~cm}^{-2}$. Both the switch-ON and -OFF times of the detector were $\approx 500 \mathrm{~ms}$. b) Photostability of the $\mathrm{Mo}_{2} \mathrm{CT}_{x}$ thin film photodetectors under continuous illumination of $660 \mathrm{~nm}$ light in ambient conditions. The device maintained $\approx 80 \%$ of the initial photocurrent even after $\approx 12000 \mathrm{~s}$. c) Ratios of the photocurrent, under $660 \mathrm{~nm}$ light illumination, with respect to the dark current as a function of storage time. d) Photocurrent and dark current ratio as a function of the bending radius. e) Ratios of the photocurrent to the dark current of the photodetectors as a function of the number of bending cycles at a bending radius of $\approx 3 \mathrm{~mm}$. Devices worked properly after 500 bending cycles at a bending radius of $\approx 3 \mathrm{~mm}$, without any significant deterioration of the performance. All of the values were obtained at a wavelength of $660 \mathrm{~nm}$ with a light intensity of $0.22 \mathrm{~W} \mathrm{~cm}$.

femtosecond visible transient absorption measurements, we probed the ultrafast dynamics of the photoactive carriers supported by $\mathrm{Mo}_{2} \mathrm{CT}_{x}$ nanosheets. The demonstrated ability of coupling with light and dephasing of SPs with a short lifetime have led to a photoresponse outperforming that of photoelectron-based semiconductor devices. Our findings shed light on the knowledge of photocurrent generation mechanisms in MXenes, making them much more viable for many photonic and plasmonic device applications.

\section{Experimental Section}

Experimental details including various MAX and MXene syntheses, device fabrication, and several characterizations can be found in the Supporting Information.

\section{Supporting Information}

Supporting Information is available from the Wiley Online Library or from the author. 


\section{Acknowledgements}

D.B.V. and J.K.E. contributed equally to this work. Research reported in this publication was supported by the King Abdullah University of Science and Technology (KAUST). The authors thank Dr. Rajeshkumar Mohanaraman for his help in the MAX phase synthesis. The authors also thank Fangwang Ming for his help with the XRD measurements and Qui Jiang for several useful discussions.

\section{Conflict of Interest}

The authors declare no conflict of interest.

\section{Keywords}

flexible photodetectors, low-frequency Raman spectroscopy, molybdenum carbide, MXenes, surface plasmons

Received: November 27, 2018

Revised: May 29, 2019

Published online:

[1] M. Naguib, M. Kurtoglu, V. Presser, J. Lu, J. Niu, M. Heon, L. Hultman, Y. Gogotsi, M. W. Barsoum, Adv. Mater. 2011, 23, 4248.

[2] B. Anasori, M. R. Lukatskaya, Y. Gogotsi, Nat. Rev. Mater. 2017, 2, 16098.

[3] M. Ghidiu, M. R. Lukatskaya, M.-Q. Zhao, Y. Gogotsi, M. W. Barsoum, Nature 2014, 516, 78.

[4] F. Shahzad, M. Alhabeb, C. B. Hatter, B. Anasori, S. Man Hong, C. M. Koo, Y. Gogotsi, Science 2016, 353, 1137.

[5] Y.-Z. Zhang, K. H. Lee, D. H. Anjum, R. Sougrat, Q. Jiang, H. Kim, H. N. Alshareef, Sci. Adv. 2018, 4, eaat0098.

[6] P. Nayak, Q. Jiang, R. Mohanraman, D. Anjum, M. N. Hedhili, H. N. Alshareef, Nanoscale 2018, 10, 17030.

[7] H. Kim, B. Anasori, Y. Gogotsi, H. N. Alshareef, Chem. Mater. 2017, 29, 6472

[8] L. Ding, Y. Wei, L. Li, T. Zhang, H. Wang, J. Xue, L.-X. Ding, S. Wang, J. Caro, Y. Gogotsi, Nat. Commun. 2018, 9, 155.

[9] K. Maleski, V. N. Mochalin, Y. Gogotsi, Chem. Mater. 2017, 29, 1632.

[10] O. Mashtalir, M. Naguib, V. N. Mochalin, Y. Dall'Agnese, M. Heon, M. W. Barsoum, Y. Gogotsi, Nat. Commun. 2013, 4, 1716.

[11] M. Naguib, V. N. Mochalin, M. W. Barsoum, Y. Gogotsi, Adv. Mater. 2014, 26, 992.

[12] Y. Dong, S. S. K. Mallineni, K. Maleski, H. Behlow, V. N. Mochalin, A. M. Rao, Y. Gogotsi, R. Podila, Nano Energy 2018, 44, 103.

[13] Q. Jiang, C. Wu, Z. Wang, A. C. Wang, J.-H. He, Z. L. Wang, H. N. Alshareef, Nano Energy 2018, 45, 266.

[14] S. Chertopalov, V. N. Mochalin, ACS Nano 2018, 12, 6109.

[15] Z. Wang, H. Kim, H. N. Alshareef, Adv. Mater. 2018, 30, 1706656.

[16] Y. Dong, S. Chertopalov, K. Maleski, B. Anasori, L. Hu, S. Bhattacharya, A. M. Rao, Y. Gogotsi, V. N. Mochalin, R. Podila, Adv. Mater. 2018, 30, 1705714.

[17] A. D. Dillon, M. J. Ghidiu, A. L. Krick, J. Griggs, S. J. May, Y. Gogotsi, M. W. Barsoum, A. T. Fafarman, Adv. Funct. Mater. 2016, 26, 4162.

[18] G. Li, K. Kushnir, Y. Dong, S. Chertopalov, A. M. Rao, V. N. Mochalin, R. Podila, L. V. Titova, 2D Mater. 2018, 5, 035043.

[19] J. K. El-Demellawi, S. Lopatin, J. Yin, O. F. Mohammed, H. N. Alshareef, ACS Nano 2018, 12, 8485.

[20] V. Mauchamp, M. Bugnet, E. P. Bellido, G. A. Botton, P. Moreau, D. Magne, M. Naguib, T. Cabioc'h, M. W. Barsoum, Phys. Rev. B 2014, 89, 235428.
[21] K. Chaudhuri, M. Alhabeb, Z. Wang, V. M. Shalaev, Y. Gogotsi, A. Boltasseva, ACS Photonics 2018, 5, 1115.

[22] X. Jiang, S. Liu, W. Liang, S. Luo, Z. He, Y. Ge, H. Wang, R. Cao, F. Zhang, Q. Wen, J. Li, Q. Bao, D. Fan, H. Zhang, Laser Photonics Rev. 2018, 12, 1700229.

[23] F. Xia, T. Mueller, Y.-M. Lin, A. Valdes-Garcia, P. Avouris, Nat. Nanotechnol. 2009, 4, 839.

[24] Z. Yin, H. Li, H. Li, L. Jiang, Y. Shi, Y. Sun, G. Lu, Q. Zhang, X. Chen, H. Zhang, ACS Nano 2012, 6, 74.

[25] G. V. Hartland, L. V. Besteiro, P. Johns, A. O. Govorov, ACS Energy Lett. 2017, 2, 1641.

[26] S. Adachi, Optical Properties of Crystalline and Amorphous Semiconductors, Springer, Boston, MA, 1999.

[27] J. Halim, S. Kota, M. R. Lukatskaya, M. Naguib, M.-Q. Zhao, E. J. Moon, J. Pitock, J. Nanda, S. J. May, Y. Gogotsi, M. W. Barsoum, Adv. Funct. Mater. 2016, 26, 3118.

[28] Z. W. Seh, K. D. Fredrickson, B. Anasori, J. Kibsgaard, A. L. Strickler, M. R. Lukatskaya, Y. Gogotsi, T. F. Jaramillo, A. Vojvodic, ACS Energy Lett. 2016, 1, 589.

[29] C. C. Lai, R. Meshkian, M. Dahlqvist, J. Lu, L. Å. Näslund, O. Rivin, E. N. Caspi, O. Ozeri, L. Hultman, P. Eklund, M. W. Barsoum, J. Rosen, Acta Mater. 2015, 99, 157.

[30] J. Halim, K. M. Cook, M. Naguib, P. Eklund, Y. Gogotsi, J. Rosen, M. W. Barsoum, Appl. Surf. Sci. 2016, 362, 406.

[31] J. Baltrusaitis, B. Mendoza-Sanchez, V. Fernandez, R. Veenstra, N. Dukstiene, A. Roberts, N. Fairley, Appl. Surf. Sci. 2015, 326, 151.

[32] K. Inzani, M. Nematollahi, F. Vullum-Bruer, T. Grande, T. W. Reenaas, S. M. Selbach, Phys. Chem. Chem. Phys. 2017, 19, 9232.

[33] A. Borgschulte, O. Sambalova, R. Delmelle, S. Jenatsch, R. Hany, F. Nüesch, Sci. Rep. 2017, 7, 40761.

[34] C. R. Clayton, Y. C. Lu, Surf. Interface Anal. 1989, 14, 66.

[35] J. Światowska-Mrowiecka, S. de Diesbach, V. Maurice, S. Zanna, L. Klein, E. Briand, I. Vickridge, P. Marcus, J. Phys. Chem. C 2008, 112, 11050.

[36] M. Rouhani, Y. L. Foo, J. Hobley, J. Pan, G. S. Subramanian, X. Yu, A. Rusydi, S. Gorelik, Appl. Surf. Sci. 2013, 273, 150.

[37] S. Bandaru, G. Saranya, N. J. English, C. Yam, M. Chen, Sci. Rep. 2018, 8, 10144

[38] S. Balendhran, J. Deng, J. Z. Ou, S. Walia, J. Scott, J. Tang, K. L. Wang, M. R. Field, S. Russo, S. Zhuiykov, M. S. Strano, N. Medhekar, S. Sriram, M. Bhaskaran, K. Kalantar-Zadeh, Adv. Mater. 2013, 25, 109.

[39] G. E. Buono-Core, A. H. Klahn, C. Castillo, E. Muñoz, C. Manzur, G. Cabello, B. Chornik, J. Non-Cryst. Solids 2014, 387, 21.

[40] D. V. Pham, R. A. Patil, J.-H. Lin, C.-C. Lai, Y. Liou, Y.-R. Ma, Nanoscale 2016, 8, 5559.

[41] A. Bouzidi, N. Benramdane, H. Tabet-Derraz, C. Mathieu, B. Khelifa, R. Desfeux, Mater. Sci. Eng., B 2003, 97, 5.

[42] X. Gong, M. Tong, Y. Xia, W. Cai, J. S. Moon, Y. Cao, G. Yu, C.-L. Shieh, B. Nilsson, A. J. Heeger, Science 2009, 325, 1665.

[43] D. B. Velusamy, M. A. Haque, M. R. Parida, F. Zhang, T. Wu, O. F. Mohammed, H. N. Alshareef, Adv. Funct. Mater. 2017, 27, 1605554.

[44] N. Huo, G. Konstantatos, Adv. Mater. 2018, 30, 1801164.

[45] O. Lopez-Sanchez, D. Lembke, M. Kayci, A. Radenovic, A. Kis, Nat. Nanotechnol. 2013, 8, 497.

[46] A. S. Rubio, Modified Au-Based Nanomaterials Studied by Surface Plasmon Resonance Spectroscopy, Springer, Cham, 2015.

[47] P. Narang, R. Sundararaman, A. Atwater Harry, Nanophotonics 2016, 5, 96

[48] M. L. Brongersma, N. J. Halas, P. Nordlander, Nat. Nanotechnol. 2015, 10, 25.

[49] W. Li, G. Valentine Jason, Nanophotonics 2017, 6, 177. 
[50] E. Cortés, W. Xie, J. Cambiasso, A. S. Jermyn, R. Sundararaman, P. Narang, S. Schlücker, S. A. Maier, Nat. Commun. 2017, 8, 14880.

[51] D. B. Velusamy, R. H. Kim, S. Cha, J. Huh, R. Khazaeinezhad, S. H. Kassani, G. Song, S. M. Cho, S. H. Cho, I. Hwang, J. Lee, K. Oh, H. Choi, C. Park, Nat. Commun. 2015, 6, 8063.

[52] M. M. Furchi, D. K. Polyushkin, A. Pospischil, T. Mueller, Nano Lett. 2014, 14, 6165.
[53] J. Zou, Q. Zhang, K. Huang, N. Marzari, J. Phys. Chem. C 2010, 114, 10725.

[54] Z. Ling, C. E. Ren, M.-Q. Zhao, J. Yang, J. M. Giammarco, J. Qiu, M. W. Barsoum, Y. Gogotsi, Proc. Natl. Acad. Sci. USA 2014, 111, 16676.

[55] F. H. L. Koppens, T. Mueller, P. Avouris, A. C. Ferrari, M. S. Vitiello, M. Polini, Nat. Nanotechnol. 2014, 9, 780. 